\title{
Gamma-rays from harmonically resonant betatron oscillations in a plasma wake
}

\author{
Silvia Cipiccia', Mohammad R. Islam¹, Bernhard Ersfeld', Richard P. Shanks', Enrico Brunetti', \\ Gregory Vieux', Xue Yang${ }^{1}$, Riju C. Issac ${ }^{1}$, Samuel M. Wiggins ${ }^{1}$, Gregor H. Welsh', Maria-Pia Anania', \\ Dzmitry Maneuski ${ }^{2}$, Rachel Montgomery ${ }^{2}$, Gary Smith², Matthias Hoek ${ }^{2}$, David J. Hamilton ${ }^{2}$, \\ Nuno R. C. Lemos ${ }^{3}$, Dan Symes ${ }^{4}$, Pattathil P. Rajeev ${ }^{4}$, Val O. Shea ${ }^{2}$, João M. Dias ${ }^{3}$ \\ and Dino A. Jaroszynski ${ }^{1 \star}$
}

\begin{abstract}
An intense laser pulse in a plasma can accelerate electrons ${ }^{1-4}$ to $\mathrm{GeV}$ energies in centimetres ${ }^{5-7}$. Transverse betatron motion ${ }^{8,9}$ in the plasma wake results in X-ray photons with an energy that depends on the electron energy, oscillation amplitude and frequency of the betatron motion ${ }^{10-12}$. Betatron X-rays from laser-accelerator electrons have hitherto been limited to spectra peaking between 1 and $10 \mathrm{keV}$ (ref. 13). Here we show that the betatron amplitude is resonantly enhanced when electrons interact with the rear of the laser pulse ${ }^{14,15}$. At high electron energy, resonance occurs when the laser frequency is a harmonic of the betatron frequency, leading to a significant increase in the photon energy. $10^{8}$ gammaray photons, with spectra peaking between 20 and $150 \mathrm{keV}$, and a peak brilliance $>10^{23}$ photons $\mathrm{s}^{-1} \mathrm{mrad}^{-2} \mathrm{~mm}^{-2}$ per $0.1 \%$ bandwidth, are measured for $700 \mathrm{MeV}$ beams, with $10^{7}$ photons emitted between 1 and $7 \mathrm{MeV}$. Femtosecond duration gamma-rays may find uses in imaging, isotope production, probing dense matter, homeland security and nuclear physics ${ }^{16}$.

$\mathrm{X}$-ray pulses from synchrotron sources have become immensely useful tools for investigating the structure of matter ${ }^{17}$, which has led to a huge international effort to construct light sources for many different scientific and technological applications. Synchrotrons are usually based on radio-frequency (RF) accelerating cavities that are limited to fields of 10-100 $\mathrm{MV} \mathrm{m}^{-1}$ because of electrical breakdown, which results in very large and expensive devices.
\end{abstract}

High-power lasers, on the other hand, have led to the development of many new areas of science, as diverse as inertial confinement fusion and laboratory astrophysics to the study of warm dense matter. However, they now have the potential to transform accelerator and light source technology. In the late 1970s, Tajima and Dawson ${ }^{1}$ proposed harnessing the ponderomotive force associated with intense laser fields to excite plasma waves and form wake-like structures ${ }^{18}$ (as behind a boat) that travel with a velocity close to the speed of light, $c$. The electrostatic forces of these charge density structures can rapidly accelerate particles to very high energies ${ }^{6}$; where momentum is gained analogous to a surfer riding an ocean wave. Recent progress in the development of laser wakefield accelerators (LWFAs) has enabled electron beams to be accelerated with unprecedented acceleration gradients ${ }^{2-4}$, three orders of magnitude higher than in RF cavities, thus reducing a $100 \mathrm{~m}$ long GeV accelerator to centimetres in length ${ }^{6}$. The LWFA can now produce high-quality electron beams with low emittance, $\varepsilon_{n}$, of the order $1 \pi \mathrm{mm} \mathrm{mrad}^{19}$, small energy spread ${ }^{20}, \delta \gamma / \gamma \ll 1 \%$, where $\gamma$ is the Lorenz factor, and high charge ${ }^{4}, Q=10-100 \mathrm{pC}$. At high laser intensities, in the so-called blowout regime ${ }^{21}$, the LWFA structure has an approximately spherical bubble shape with a radius of $R \approx 2 \sqrt{a_{0}} c / \omega_{\mathrm{p}}$, which is primarily determined by the normalized laser vector potential, $a_{0}=e A / m_{e} c^{2}$ and the plasma frequency, $\omega_{\mathrm{p}}=\sqrt{4 \pi n_{\mathrm{p}} e^{2} / m_{e}}$, where $n_{p}$ is the plasma density, $e$, the electron charge and $m_{e}$, the electron mass ${ }^{22}$. The plasma wave is efficiently driven when the laser pulse duration is approximately a plasma period. Micrometre-long electron bunches that extend only a fraction of the plasma wavelength, $\lambda_{\mathrm{p}}=2 \pi c / \omega_{\mathrm{p}}$, are self-injected and accelerated $^{5,22}$, which leads to peak currents of kiloamperes ${ }^{3}$. This makes the LWFA an attractive candidate for driving compact synchrotron and free-electron laser ${ }^{16}$ radiation sources. As a first step towards developing a compact source, synchrotron emission from an undulator driven by a LWFA has been demonstrated ${ }^{23}$. However, the confining force of the bubble structure can also act as an effective wiggler to produce X-ray radiation peaking in the sub-ångström range $\mathrm{e}^{10}$.

The electrostatic fields inside the plasma bubble ${ }^{21}$ give rise to very large forces. For a laser spot equal to the plasma bubble diameter, the laser energy is depleted over a length $L_{p d}=\gamma_{g}^{2} c \tau_{l}$, where $\gamma_{g}=\omega_{0} / \omega_{\mathrm{p}}$ is the relativistic factor associated with the laser group velocity ${ }^{22}$. Typically, when $a_{0} \geq 3$, electrons are injected into the bubble from the background plasma ${ }^{2-4}$ and accelerate until they reach the dephasing length ${ }^{22}, L_{d}=4 c \gamma_{g}^{2} \sqrt{a_{0}} / 3 \omega_{\mathrm{p}}$. At the dephasing point the energy gained is $\gamma_{d} \approx 2 \gamma_{g}^{2} a_{0} / 3$. The restoring force of the bubble results in harmonic oscillations of electrons in the bubble at a characteristic betatron frequency, $\omega_{\beta}=\omega_{\mathrm{p}} / \sqrt{2 \gamma}$. The conjugate variables of the oscillator are the excursion and momentum, with respective amplitudes $r_{\beta}$ and $m_{e} c a_{\beta}$, where $a_{\beta}=\gamma \beta_{\perp}=k_{\mathrm{p}} \sqrt{\gamma / 2} r_{\beta}$ is the normalized transverse momentum, with $\gamma$ the Lorentz factor, $k_{\mathrm{p}}=2 \pi / \lambda_{\mathrm{p}}$, and $\beta_{\perp}=v_{\perp} / c$ the normalized transverse velocity. As in a conventional wiggler, radiation is emitted within a half-angle $\varphi=a_{\beta} / \gamma$, as a series of harmonics $\omega_{h}=h 2 \bar{\gamma}_{z}^{2} \omega_{\beta}$, where $\bar{\gamma}_{z}=\gamma(1+$ $\left.a_{\beta}^{2} / 2\right)^{-1 / 2}$ is the average longitudinal Lorentz factor and $h$ is the harmonic number. When $a_{\beta} \geq 1$, the longitudinal Lorentz factor is modulated at even harmonics of $\omega_{\beta}$ and the spectrum contains many merged harmonics with a critical harmonic at $h_{\mathrm{c}} \approx 3 a_{\beta}^{3} / 8$, giving $E_{\mathrm{c}}=\hbar \omega_{\mathrm{c}}=h_{\mathrm{c}} \hbar 2 \bar{\gamma}_{z}^{2} \omega_{\beta}$, which is close to the synchrotron

\footnotetext{
${ }^{1}$ University of Strathclyde, Department of Physics, Scottish Universities Physics Alliance, John Anderson Building, 107 Rottenrow, Glasgow, G4 ONG, UK,

${ }^{2}$ University of Glasgow, Department of Physics and Astronomy, Scottish Universities Physics Alliance, Glasgow, G12 8QQ, UK, ${ }^{3}$ GoLP/Instituto de Plasmas e Fusão Nuclear, Instituto Superior Técnico, Avenida Rovisco Pais 1049-001, Lisbon, Portugal, ${ }^{4}$ Central Laser Facility, Science and Technology Facilities Council, Rutherford Appleton Laboratory, Harwell Science and Innovation Campus, Didcot, OX11 0QX, UK. *e-mail: d.a.jaroszynski@strath.ac.uk.
} 

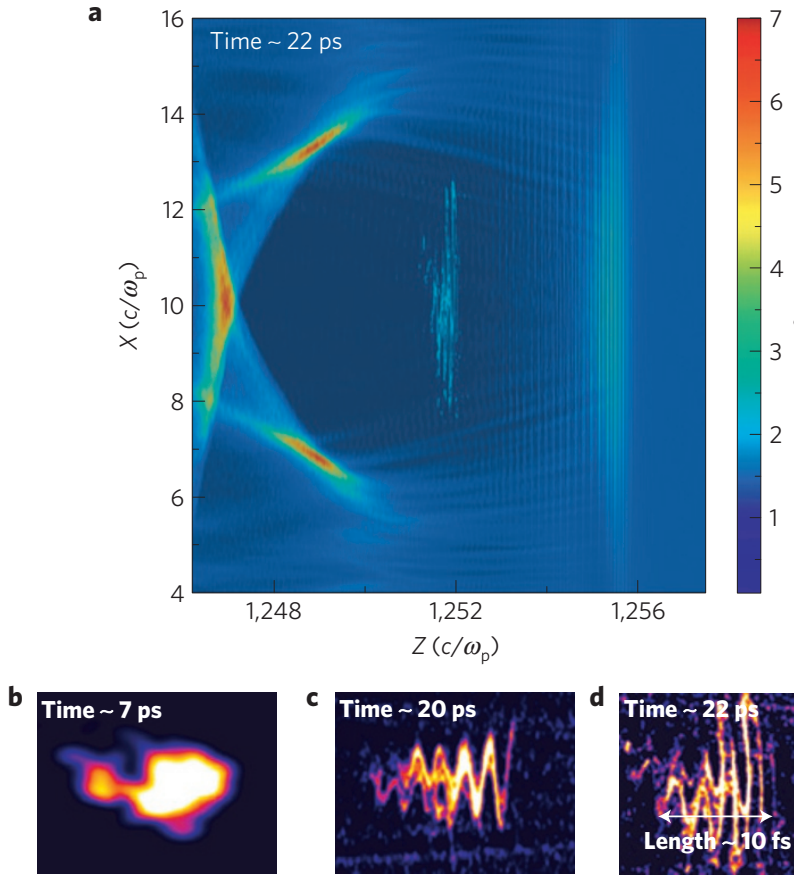
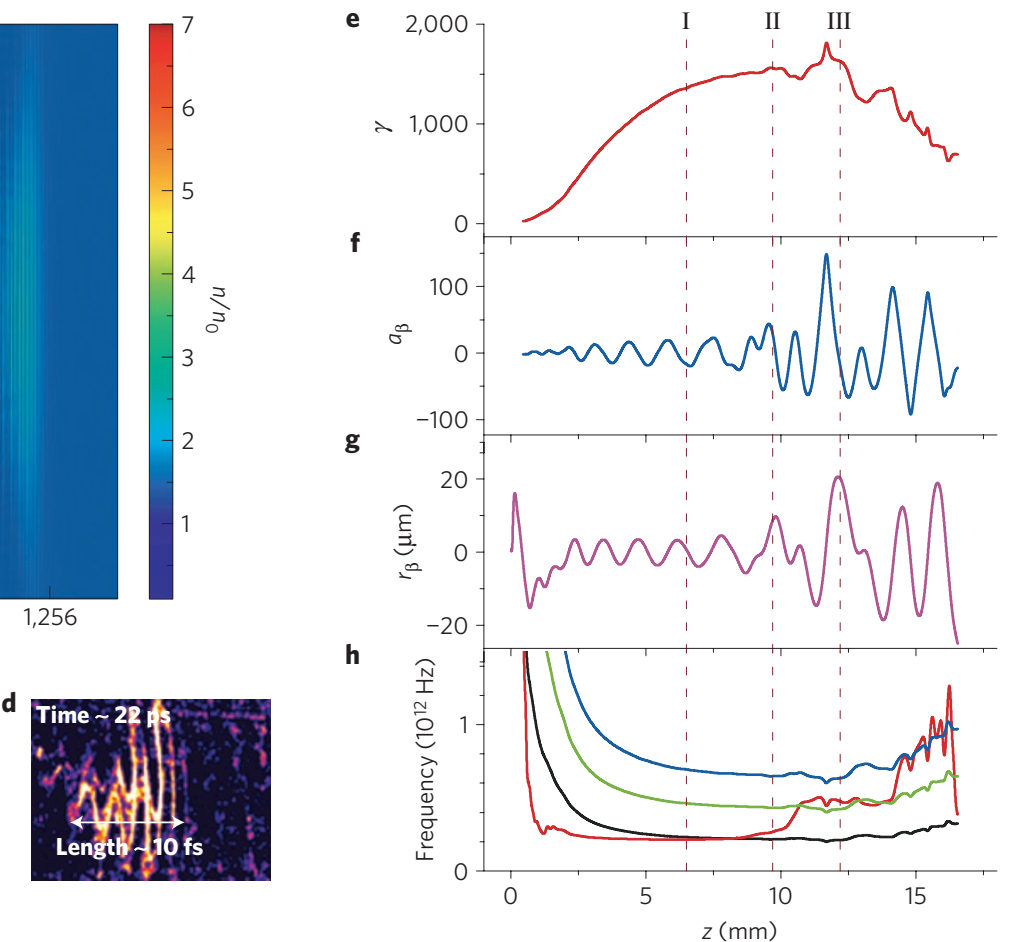

Figure 1 | OSIRIS simulations of laser-driven betatron oscillations. a, Snapshot of the electron distribution. b-d, Electron beam trajectories for the strongly damped (b), weakly resonant (c) and strongly resonant (d) case. e-h, The evolutions, for a selected trajectory, are shown of $\gamma(\mathbf{e}), a_{\beta}$, (f), $r_{\beta}(\mathbf{g})$, and the first (black line), second (green line) and third harmonics (blue line) of $\omega_{\beta}$, and $\tilde{\omega}_{0}$, the Doppler-shifted laser frequency as seen by the electrons (red line) (h). The three vertical dashed lines indicate the different regimes that can occur with almost the same $\gamma$ : strongly damped case (I), weakly resonant case (II), strongly resonant case (III). Parameters used in simulation: $n_{e}=1.8 \times 10^{18} \mathrm{~cm}^{-3} \lambda=800 \mathrm{~nm}$, laser spot size $35 \mu \mathrm{m}$, laser pulse duration $70 \mathrm{fs}$ and initially $a_{0}=3$. The normalized transverse momentum varies from $a_{\beta}=5.6$ for $r_{\beta}=1 \mu \mathrm{m}$ to $a_{\beta}=150$ for $r_{\beta}=20 \mu \mathrm{m}$, at $\gamma=1,600$. The laser field, with frequency $\omega_{0}{ }^{\prime}$ and phase velocity $v_{p}$, acts on electrons co-moving with longitudinal velocity $v_{z}$, at the downshifted frequency $\tilde{\omega}$. Efficient energy transfer from laser to betatron motion requires resonance (that is, $\tilde{\omega} \approx 1 \omega_{\beta}$, where $/$ is the betatron harmonic number).

critical energy, $E_{\mathrm{c}} \approx 3 \hbar \omega_{\mathrm{p}}^{2} r_{\beta} \gamma^{2} / 2 c$ (ref. 24). The radiation rate, determined from the Lienard-Wiechert potentials ${ }^{24}$, scales with $\gamma^{2}$, and $N_{\text {phot }}=(2 \pi / 9) \alpha_{f} a_{\beta}$ photons per electron are emitted every betatron oscillation cycle, where $\alpha_{f}=1 / 137$ is the fine structure constant. To obtain a high photon flux and high photon energies the energy and betatron amplitude should be maximized for a given $\omega_{\mathrm{p}}$.

The presence of the laser field inside the bubble can drastically change the electron dynamics ${ }^{25}$. When the laser partially fills the bubble $\left(\tau_{l} \geq R / c\right)$ it can interact with the electrons. As the average velocity of oscillating electrons increases, owing to acceleration by the longitudinal force of the bubble, they see a continually evolving Doppler-downshifted frequency of the laser radiation. The electrons oscillate anharmonically because of the nonlinear relationship between velocity and momentum. This allows them to be resonantly driven at harmonics of the betatron frequency. For linearly polarized laser radiation, this interaction can be described by the driven nonlinear oscillator equation $\ddot{y}+\Gamma \dot{y}+\omega_{\beta}^{2} y=F_{L y} /(m \gamma)$, where $\Gamma=\dot{\gamma} / \gamma$ is the damping rate due to the relativistic mass increase and $F_{L y}=e(\mathrm{~d} A / \mathrm{d} t-\dot{y} \partial A / \partial y)$ is the Lorentz force. The $\gamma$-dependence of $\omega_{\beta}$ leads to a shift of the resonance on a slow timescale, and anharmonic motion on a fast timescale. The laser frequency can be shifted by several per cent to $\omega_{0}^{\prime}$ by photon deceleration ${ }^{26}$ and is Doppler-shifted to $\tilde{\omega}=\omega_{0}^{\prime}\left(1 /\left(2 \bar{\gamma}_{z}^{2}\right)+1 /\left(2 \gamma_{g}^{\prime 2}\right)\right)$, where $\gamma_{g}^{\prime}$ is the Lorentz factor for the group velocity of the segment of the laser pulse interacting with the electrons in the bubble. The oscillation amplitude grows rapidly when $\Gamma \ll \omega_{\beta}$ and the laser frequency is in resonance with a harmonic of the betatron frequency, $\tilde{\omega}=l \omega_{\beta}$, where $l$ is the harmonic number. $\Gamma$ vanishes at dephasing $\left(z \simeq L_{d}\right)$, when $\dot{\gamma} \rightarrow 0$, causing the betatron amplitude to increase suddenly. At this point both $a_{\beta}$ and $\gamma$ are at their maximum. Harmonically resonant betatron motion is clearly observed in numerical simulations using the OSIRIS particle in cell (PIC) $\operatorname{code}^{27}$, for our experimental parameters, as shown in Fig. 1a. Electrons that are injected at the rear of the 'bubble' initially perform independent betatron oscillations arising from their initial transverse position and momentum (Fig. 1b). The amplitude of these 'free' oscillations are relativistically damped owing to the longitudinal acceleration (that is, $\Gamma>0$ ). When resonance occurs (that is, $\Gamma \approx 0$ and phase $\simeq \pi$ ) the betatron amplitude grows rapidly until $r_{\beta} \approx R$, as shown in Fig. 1g. As the laser phase is the same for all the electrons at a given longitudinal position, these forced oscillations have identical phase and the trajectories coalesce ${ }^{14}$, as shown in the well-defined oscillations evident in Fig. 1c,d. The increase in the transverse momentum $a_{\beta}$ causes the Doppler-shifted laser frequency to drift into resonance with harmonics of $\omega_{\beta}$ (as observed in Fig. 1h). Electrons can be driven at harmonics of the betatron frequency because of anharmonic motion arising from the nonlinear velocity-momentum relationship. They eventually begin to decelerate and leave the plasma at an arbitrary phase of the high-amplitude oscillation, with only a residual phase space spread, that is the emittance is conserved from the original 'free' oscillation and is similar to that just after injection, whereas the high-amplitude oscillation of the transverse momentum leads to shot-to-shot pointing variations.

In a realistic experiment, electron injection is a highly nonlinear process that depends on both the laser and plasma properties ${ }^{5,22,26}$. As a result the injection point and the net acceleration length can vary. Thus, for a fixed plasma medium length, the wakefield acceleration structure can vanish either before dephasing (I-illustrated in Fig. 1), at dephasing (II) or beyond dephasing (III). Close to 


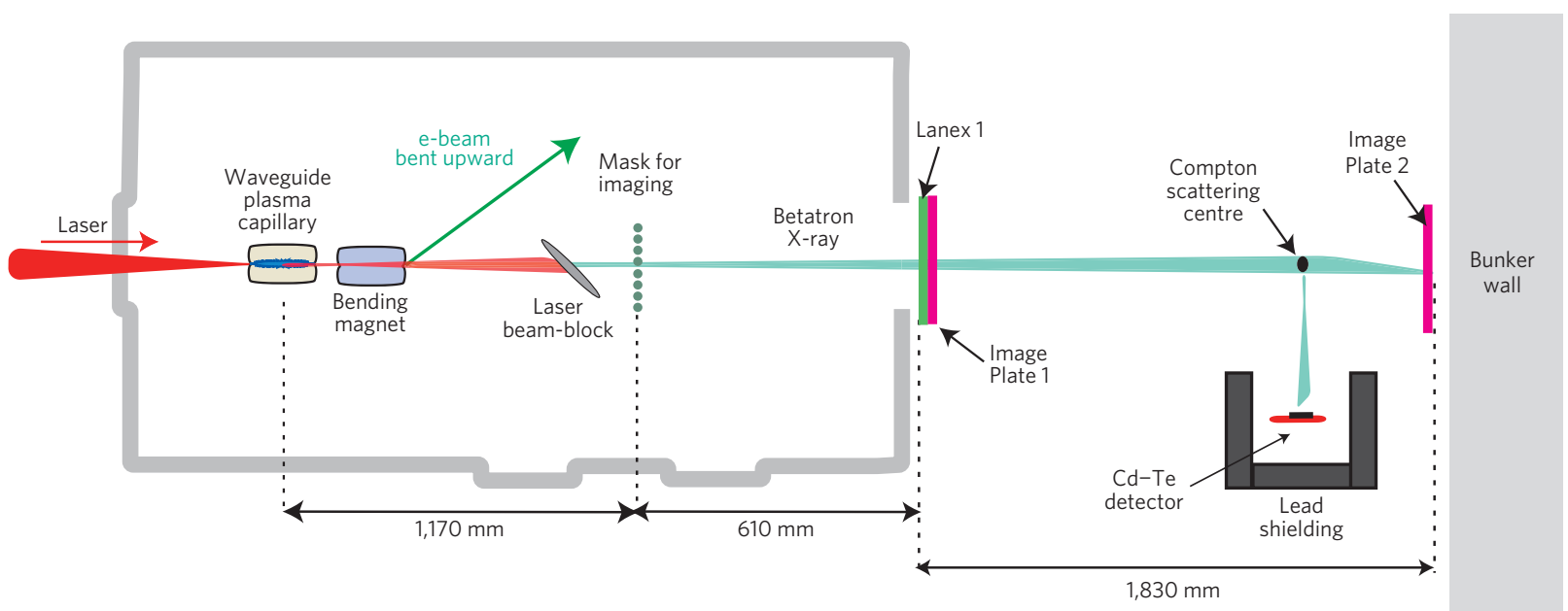

Figure 2 | Experimental set-up. A F/16 spherical mirror focuses the laser pulse ( $5 \mathrm{~J}, 55 \pm 5 \mathrm{fs}, 800 \mathrm{~nm}$ ) to a $40 \pm 5 \mu \mathrm{m}$ diameter spot at the entrance of the $4 \mathrm{~cm}$ long, $300 \mu \mathrm{m}$ diameter, pre-formed plasma capillary waveguide ${ }^{29}$ with an on-axis density of $n_{\mathrm{p}} \approx 1.8-2.0 \times 10^{18} \mathrm{~cm}^{-3}$. The laser beam initially has an intensity of $9 \times 10^{18} \mathrm{~W} \mathrm{~cm}^{-2}$, which corresponds to $a_{0}=2$ and a bubble radius $R=10 \mu \mathrm{m}$. A $600 \mu \mathrm{m}$ thick Al foil blocks laser light from X-ray detectors. Lanex 1 and Image Plate 1 are used to measure the high-energy portion of the electron spectra simultaneously with the X-ray beam profiles (dipole field set to $0.7 \mathrm{~T}$ ) or the electron beam profile alone (dipole field set to zero). Image Plate 2, placed on axis, records the X-ray profile, phase contrast images and, with cut-off filters, the spectra. The CdTe detector, placed in a screened enclosure, records Compton side-scattered radiation. For further details see the Methods section.

a

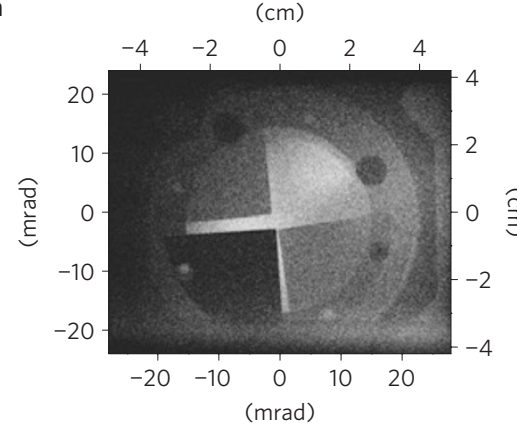

b

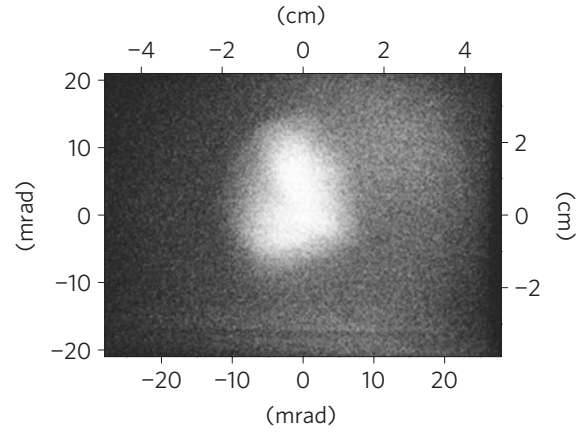

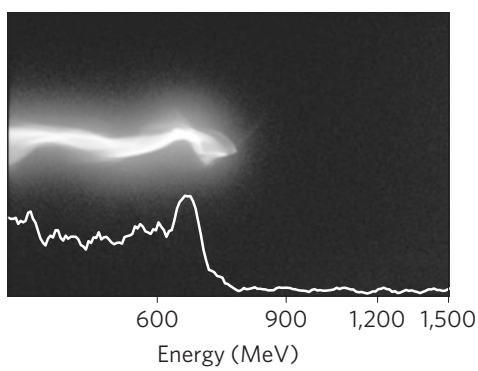

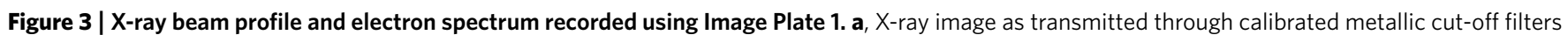
( $0.3 \mathrm{~mm}$ (top left) and $1 \mathrm{~mm}$ (bottom left) tungsten foils, $0.5 \mathrm{~mm}$ (top right) and $1 \mathrm{~mm}$ (bottom right) copper foils). The calibrated cut-off filters give a spectral peak at $20 \mathrm{keV}\left(E_{\mathrm{c}} \approx 60 \pm 15 \mathrm{keV}\right)$. b, X-ray beam profile used to deduce a divergence of 14 mrad, measured simultaneously with the 700 MeV electron beam shown in c.

dephasing, $r_{\beta}$ and $a_{\beta}$ can grow substantially without an appreciable change in $\gamma$, as can be seen in Fig. 1 . This accounts for the variability of the electron beam properties of the LWFA.

To investigate the potential of betatron emission as a brilliant source of gamma-rays, we have focussed a $5 \mathrm{~J}, 55 \pm 5$ fs duration laser pulse from the ASTRA-Gemini laser ${ }^{28}$ into a preformed plasma waveguide ${ }^{16,29}$ with $n_{p} \approx 2 \times 10^{18} \mathrm{~cm}^{-3}$, as shown in Fig. 2 . The initial vector potential $a_{0} \simeq 2$ is enhanced as a result of the nonlinear evolution of the laser fields and can easily treble in value, which leads to self-injection and quasi-monoenergetic electron bunches.

Resonant betatron emission has been investigated by simultaneously monitoring the electron beam properties using an electron energy spectrometer, LANEX (ref. 30) screens and image plates ${ }^{31}$, and X-ray photon properties, using image plates and/or a semiconductor X-ray detector. During the investigation, three distinct sets of data (corresponding to regions I, II and III in Fig. 1) have been recorded. In all cases the electron energy was around $700 \mathrm{MeV}$. In the first experiment, carried out at a density of $\approx 1.6 \times 10^{18} \mathrm{~cm}^{-3}$ (obtained with $50 \mathrm{mbar}$ gas pressure in the capillary waveguide), we studied the highly damped $(\Gamma \gg 0)$ stage (I) by determining the photon energy peak and angular divergence. By increasing the density to $\approx 2 \times 10^{18} \mathrm{~cm}^{-3}$ (obtained with 60 mbar gas pressure in the capillary waveguide) the dephasing length is reduced, thus allowing the weakly and strongly resonant regimes (II and III, where $\Gamma \approx 0$ ) to be investigated.

$\mathrm{X}$-rays emitted in the highly damped stage (I) have spectra centred around $20 \mathrm{keV}$, which precluded the use of the CdTe detector $^{32}$ because of strong $\mathrm{K} \alpha$ emission at $30 \mathrm{keV}$ from the semiconductor bulk. As an alternative, a set of copper and tungsten cut-off filters has been used to selectively attenuate the X-ray flux and estimate the spectral content, which is then correlated with the electron spectra. The spectra peak around $20 \mathrm{keV}$, which corresponds to a critical photon energy of $50-60 \mathrm{keV}$, and the X-ray beam has an angular divergence $\approx 14 \mathrm{mrad}$, as shown in Fig. 3 . This is consistent with $n_{e} \simeq 1.6 \times 10^{18} \mathrm{~cm}^{-3}, r_{\beta} \simeq 3 \mu \mathrm{m}$ and $a_{\beta} \approx 20$.

Increasing the plasma density by $20 \%$ reduces the dephasing length and allows the undamped resonant regime (II and III) to be entered. X-ray spectra, shown in Fig. 4, have been recorded using a CdTe pixellated semiconductor detector, which is described in the Methods Section. The X-ray spectrum in Fig. 4 a peaks at $50 \mathrm{keV}$ ( $E_{\mathrm{c}} \approx 150 \mathrm{keV}$ ), which corresponds to the weakly resonant case (II), where injection occurs later and the electron bunch exits the potential before dephasing and growth in $r_{\beta}$ has taken place. In contrast, the peak at $150 \mathrm{keV}\left(E_{\mathrm{c}} \approx 450 \mathrm{keV}\right)$, shown in Fig. $4 \mathrm{~b}$, corresponds to 
a

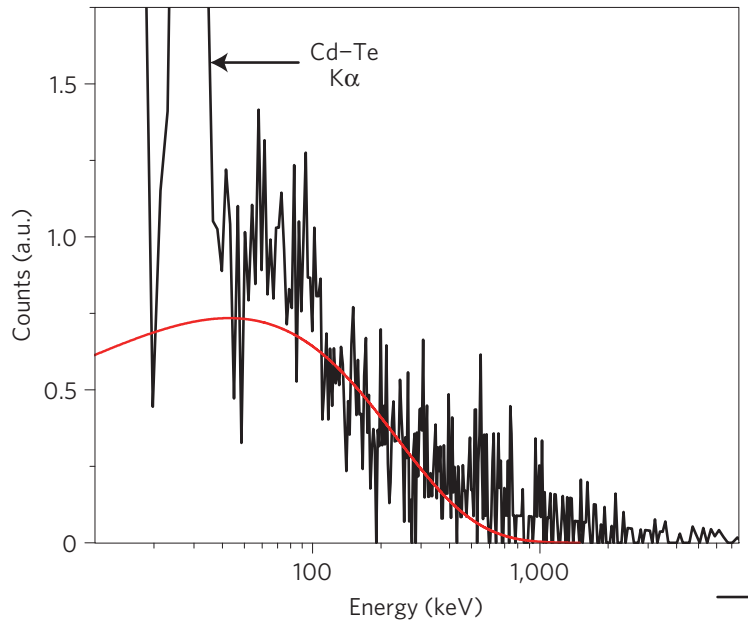

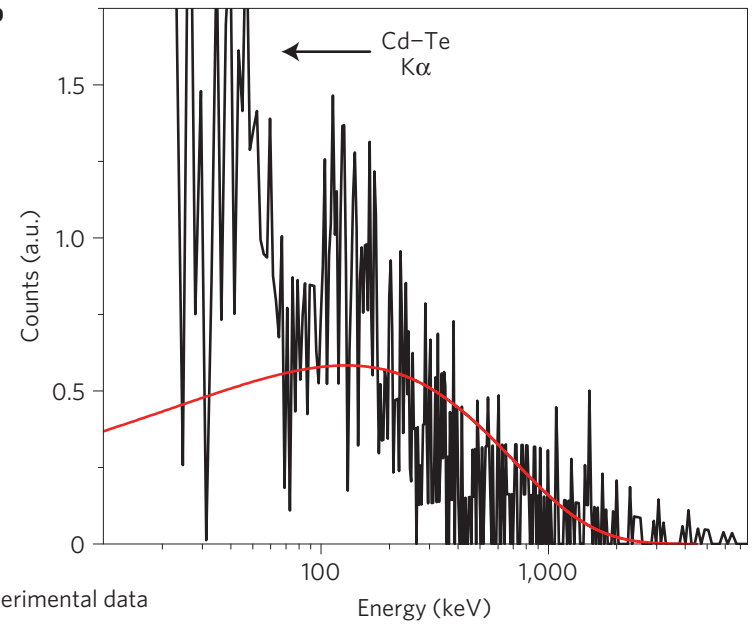

Figure 4 | Two single-shot X-ray spectra, measured with a CdTe pixellated semiconductor detector, as described in the Methods section, are shown with the predicted synchrotron spectra. a, Weakly resonant case II: spectrum peaks at $50 \mathrm{keV}\left(E_{\mathrm{c}} \approx 150 \mathrm{keV}\right)$. b. Strongly resonant case III: spectrum peaks at $150 \mathrm{keV}\left(E_{\mathrm{c}} \approx 450 \mathrm{keV}\right)$. The measured electron energy is $633 \pm 70 \mathrm{MeV}$ for both cases.

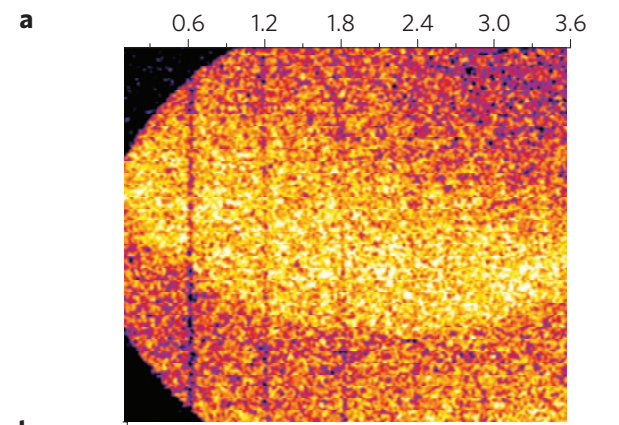

b

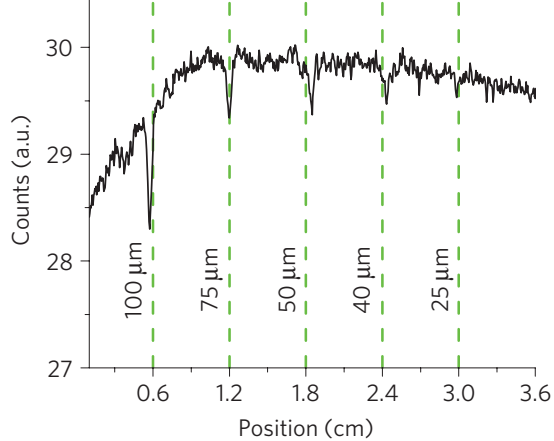

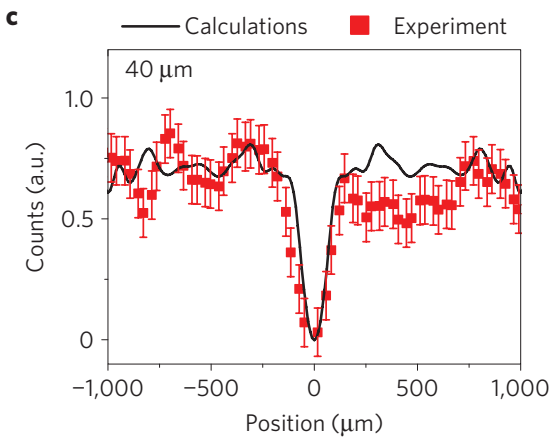

e

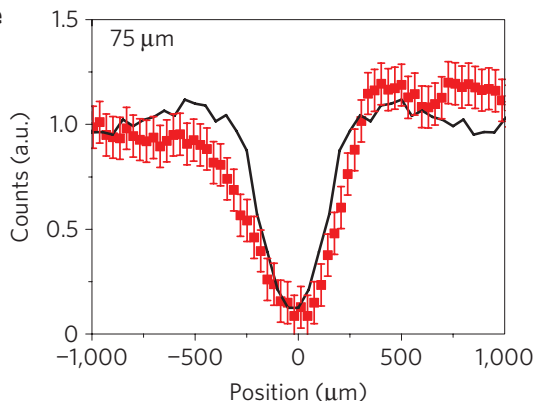

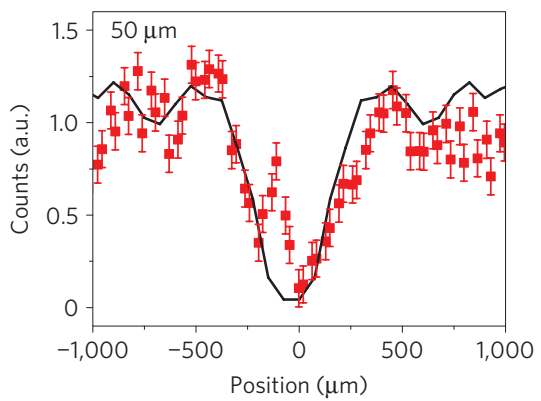

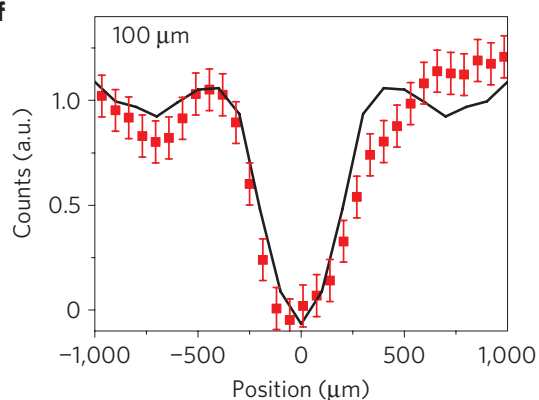

Figure 5 | Phase contrast experiment. a, Phase contrast image recorded on an image plate, showing images of four copper wires of $100,75,50$ and $40 \mu \mathrm{m}$ thickness, respectively from left to right. $\mathbf{b}$, Lineout shows profiles where the faint shadow of a fifth $25 \mu \mathrm{m}$ thick wire is just visible at position $3 \mathrm{~mm}$. c-f, Plots of the shadows cast by the wires (squares) compared with simulated data (solid lines) that are consistent with a $15 \mu \mathrm{m}$ diameter X-ray source size. Simulations assume a X-ray spectrum ranging from 10 to $100 \mathrm{keV}$. The error bars are calculated from the standard deviation of the signal variation from pixel to pixel.

a strongly resonant interaction with the laser (III) and evolution that has already passed dephasing. This compares well with analytical calculations and numerical simulations using the OSIRIS PIC $\operatorname{code}^{27}$, which give $r_{\beta}=20 \mu \mathrm{m}, a_{\beta}=150, n_{e}=2 \times 10^{18} \mathrm{~cm}^{-3}$ and $\gamma=1,600$, shown in Fig. 1. Electrons radiate very high energy photons at the dephasing point and are then decelerated again. In both cases (II and III) the average electron energy was $630 \pm 70 \mathrm{MeV}$.

To prove that the gamma-rays originated from a source with a size equal to the betatron amplitude we have projected phasecontrast shadow images of fine wires onto an X-ray image plate, as shown in Fig. 5. By comparing the measured images from five different thickness wires, from 20 to $100 \mu \mathrm{m}$ in diameter, with those predicted for different source sizes we have found that a $15 \mu \mathrm{m}$ source produces consistent phase contrast images for all wires. This source size corresponds to a betatron amplitude $r_{\beta}$ of $7.5 \mu \mathrm{m}$, which is in good agreement with that predicted by OSIRIS for the weakly resonant case (II) and gives a value of $a_{\beta}=50$.

The betatron motion of very energetic electrons results in an intense narrow beam of X-rays, which will be polarized predominantly in the plane of betatron motion. The X-ray pulse length is estimated to be $\approx 10$ fs from simulations, shown in Fig. $1 d$, which is a reasonable assumption because the greatest number of high-energy photons are emitted when $a_{\beta}$ is large at resonance. From the X-ray detection efficiency we have estimated the number 
of emitted photons to be about $5 \times 10^{8}$. By determining the source size from phase contrast images of thin wires, a peak brilliance of $10^{23}$ photons $\mathrm{s}^{-1} \mathrm{mrad}^{-2} \mathrm{~mm}^{-2}$ per $0.1 \%$ bandwidth has been estimated. The regime of operation can be chosen by varying the plasma density and channel length. In the strongly resonant regime for $\gamma>2,000$ it should be possible to produce a large flux of photons in the $\mathrm{MeV}$ range. This would also require higher laser intensities and a lower plasma density, for example $a_{0}=10$, $n_{e}=10^{18} \mathrm{~cm}^{-3}$ and $\gamma=5,500, r_{\beta}=R=34 \mu \mathrm{m}$ gives $a_{\beta} \approx 340$, which will produce photons with a critical energy $>5 \mathrm{MeV}$ with an efficiency of the order of $1 \%$ per betatron cycle. Such a source of polarized gamma-ray photons would open up new applications in high-contrast imaging of weakly absorbing and hot dense matter, time-resolved probing of the nucleus, the production of medical radio-isotopes through photo-nuclear reactions and the production of polarized elementary particles.

\section{Methods}

Two different X-ray detectors have been used in the experiments. Single-shot X-ray spectra have been measured using a single-photon multi-pixel CdTe detector ${ }^{32}$, originally developed at CERN. The detector has been calibrated using laboratory sources and its response to $\mathrm{X}$-ray radiation over a wide energy range modelled and reproduced using GEANT4 (ref. 33). As the detector was not suitable for directly measuring the entire photon spectrum, ranging from $10 \mathrm{keV}$ to more than a MeV, especially towards the higher end, it was necessary to use an indirect method to determine the spectrum. The X-ray spectra, in the weakly and strongly resonant regimes, have been measured in a single shot using the semiconductor detector to detect $90^{\circ}$ Compton side-scattered betatron radiation from a $12 \mathrm{~mm}$ diameter aluminium rod. Compton scattering downshifts the X-ray spectrum such that spectra in the $\mathrm{MeV}$ range can be detected with adequate resolution using only the $1 \mathrm{~mm}$ thick semiconductor detector, which has a range from $20 \mathrm{keV}$ to $600 \mathrm{keV}$. Moreover, the flux of the incoming radiation is reduced without requiring filters or attenuators. GEANT4 was used to estimate the properties of the synchrotron-like spectra of the X-rays striking the aluminium rod.

Phase-contrast images of fine copper wires have been recorded on X-ray image plates (FUJI-BAS-MS-2325), which consist of a layer of barium fluorohalide phosphor crystals embedded in a plastic supporting medium. When these are exposed to X-rays, secondary electrons that are produced in the interaction are trapped in lattice defects. These then emit blue light when illuminated by $640 \mathrm{~nm}$ light $^{34}$ on readout in the scanning system. The image plate is sensitive mainly to photons in the $4-80 \mathrm{keV}$ (ref. 35) range but can also detect higher energies photons with reduced sensitivity. They are widely used for X-ray imaging thanks to their excellent dynamic range, efficiency and large area ${ }^{35}$.

Simulations were carried out using the PIC code OSIRIS (ref. 27). We performed 2D PIC simulations for an incident laser pulse with a tranverse and longitudinal Gaussian shaped envelope $a_{0}(r, t)=\exp \left[-r^{2} / \sigma_{r}^{2}-t^{2} / t^{2}\right]$. The laser pulse parameters used in the simulations were $\sigma(\mathrm{FWHM})=35 \mu \mathrm{m}$, $\tau(\mathrm{FWHM})=70 \mathrm{fs}$ and a peak $a_{0}=3$. The background plasma density used for the simulations was $n_{p}=1.8 \times 10^{18} \mathrm{~cm}^{-3}$ with a linear ramp up of $100 \mu \mathrm{m}$, followed by a constant density over $2 \mathrm{~cm}$, and then a symmetric ramp down of $100 \mu \mathrm{m}$. The grid size used in the simulations was $0.005 c / \omega_{p}$ and the time step, $0.003 \times 1 / \omega_{p}$.

Received 25 February 2011; accepted 12 August 2011; published online 18 September 2011

\section{References}

1. Tajima, T. \& Dawson, J. M. Laser electron accelerator. Phys. Rev. Lett. 43, 267-270 (1979).

2. Mangles, S. P. D. et al. Electron acceleration from the breaking of relativistic plasma waves. Nature 431, 535-538 (2004).

3. Geddes, C. G. R. et al. High quality electron beams from a laser wakefield accelerator using plasma-channel guiding. Nature 431, 538-541 (2004).

4. Faure, J. et al. A laser-plasma accelerator producing monoenergetic electron beams. Nature 431, 541-544 (2004).

5. Pukhov, A. \& Meyer-Ter-Vehn, J. Laser wake field acceleration: The highly non-linear broken wave regime. Appl. Phys. B 74, 355-361 (2002).

6. Leemans, W. P. et al. GeV electron beams from a centimetre-scale accelerator. Nature Phys. 2, 696-699 (2006).

7. Clayton, C. E. et al. Self-guided laser wakefield acceleration beyond $1 \mathrm{GeV}$ using ionization-induced injection. Phys. Rev. Lett. 105, 105003 (2010).

8. Whittum, D. H. Electromagnetic-Instability of the ion-focused regime. Phys. Fluids B 4, 730-739 (1992).

9. Kostyukov, I., Kiselev, S. \& Pukhov, A. X-ray generation in an ion channel. Phys. Plasmas 10, 4818-4828 (2003).

10. Rousse, A. et al. Production of a keV X-ray beam from synchrotron radiation in relativistic laser-plasma interaction. Phys. Rev. Lett. 93, 135005 (2004).
11. Wang, S. Q. et al. X-ray emission from betatron motion in a plasma wiggler. Phys. Rev. Lett. 88, 135004 (2002).

12. Johnson, D. K. et al. Positron production by X rays emitted by betatron motion in a plasma wiggler. Phys. Rev. Lett. 97, 175003 (2006).

13. Kneip, S. et al. Bright spatially coherent synchrotron X-rays from a table-top source. Nature Phys. 6, 980-983 (2010).

14. Nemeth, K. et al. Laser-driven coherent betatron oscillation in a laser-wakefield cavity. Phys. Rev. Lett. 100, 095002 (2008).

15. Mangles, S. P. D. et al. Laser-wakefield acceleration of monoenergetic electron beams in the first plasma-wave period. Phys. Rev. Lett. 96, 215001 (2006).

16. Jaroszynski, D. A. et al. Radiation sources based on laser-plasma interactions. Phil. Trans. R. Soc. A 364, 689-710 (2006).

17. Vainshtein, B. K. Diffraction investigation of the atomic-structure of matter. Acta Crystallogr. B 47, 145-154 (1991).

18. Sprangle, P., Esarey, E. \& Ting, A. Nonlinear-interaction of intense laser-pulses in plasmas. Phys. Rev. A 41, 4463-4467 (1990).

19. Brunetti, E. et al. Low emittance, high brilliance relativistic electron beams from a laser-plasma accelerator. Phys. Rev. Lett. 105, 215007 (2010).

20. Wiggins, S. M. et al. High quality electron beams from a laser wakefield accelerator. Plasma Phys. Control. Fusion 52, 124032 (2010).

21. Pukhov, A., Gordienko, S., Kiselev, S. \& Kostyukov, I. The bubble regime of laser-plasma acceleration: Monoenergetic electrons and the scalability. Plasma Phys. Control. Fusion 46, B179-B186 (2004).

22. Lu, W., Tzoufras, M. \& Joshi, C. Generating multi-GeV electron bunches using single stage laser wakefield acceleration in a 3D nonlinear regime. Phys. Rev. 10, 061301 (2007)

23. Schlenvoigt, H. P. et al. A compact synchrotron radiation source driven by a laser-plasma wakefield accelerator. Nature Phys. 4, 130-133 (2008).

24. Jackson, J. D. Classical Electrodynamics (John Wiley, 1999).

25. Pukhov, A., Sheng, Z. M. \& Meyer-ter-Vehn, J. Particle acceleration in relativistic laser channels. Phys. Plasmas 6, 2847-2854 (1999).

26. Reitsma, A. J. W. et al. Photon kinetic modeling of laser pulse propagation in underdense plasma. Phys. Plasmas 13, 113104 (2006).

27. Fonseca, R. A. et al. in Computational Science-ICCS 2002 (eds Peter, M. A., Sloot, A. G., Hoekstra, C. J., Kenneth Tan, J. J. \& Dongarra,) 342-351 (Lecture Notes in Computer Science, Vol. 2331, Springer, 2002).

28. Hooker, C. J. et al. Conference on Lasers and Electro-Optics/Quantum Electronics and Laser Science Conference and Photonic Applications Systems Technologies, OSA Technical Digest (CD) paper JThB2 (Optical Society of America, 2008).

29. Hooker, S. M. et al. GeV plasma accelerators driven in waveguides. Plasma Phys. Control. Fusion 49, B403 (2007).

30. Glinec, Y. et al. Absolute calibration for a broad range single shot electron spectrometer. Rev. Sci. Instrum. 77, 103301 (2006).

31. Zeil, K. et al. Absolute response of Fuji imaging plate detectors to picosecond-electron bunches. Rev. Sci. Instrum. 81, 013307 (2010).

32. Llopart, X., Ballabriga, R., Campbell, M., Tlustos, L. \& Wong, W. Timepix, a $65 \mathrm{k}$ programmable pixel readout chip for arrival time, energy and/or photon counting measurements. Nucl. Instrum. Methods A 581, 485-494 (2007).

33. Agostinelli, S. et al. GEANT4-a simulation toolkit. Nucl. Instrum. Methods A 506, 250-303 (2003).

34. Paterson, I. J., Clarke, R. J., Woolsey, N. C. \& Gregori, G. Image plate response for conditions relevant to laser-plasma interaction experiments. Meas. Sci. Technol. 19, 095301 (2008).

35. Thompson, A. et al. X-ray Data Booklet 3rd edn Pub-490 Rev.3 (Lawrence Berkeley National Laboratory, 2009).

\section{Acknowledgements}

We acknowledge the support of the UK EPSRC, STFC, the Laserlab-Europe consortium and the Extreme Light Infrastructure (ELI) project. Over the past decade many current and previous members of the ALPHA-X consortium have made contributions to the project, for which we extend thanks. We also thank D. Clark and T. McCanny for their technical support, without which the project would not have been possible. The authors would like to thank the OSIRIS consortium (UCLA/IST) for the use of OSIRIS. The work of NRCL was partially supported by FCT Portugal through the grant SFRH/BD/37838/2007

\section{Author contributions}

The experiment was conceived by D.A.J. The experiments and equipment calibration were undertaken by S.C., R.P.S, E.B., G.V., X.Y., R.C.I., S.M.W., G.H.W., M-P.A., D.M., R.M., G.S., M.H., D.J.H., N.R.C.L., V.O.S., J.M.D. and D.A.J. Theoretical analysis, interpretation and simulations were carried out by M.R.I., B.E., S.C. and D.A.J. The laser and target area operators were D.S. and P.P.R. The manuscript was prepared in the main by D.A.J., S.C., B.E. and M.R.I., with the reviewing and comment input of R.C.I., S.M.W., G.H.W., N.R.C.L. and J.M.D.

\section{Additional information}

The authors declare no competing financial interests. Reprints and permissions information is available online at http://www.nature.com/reprints. Correspondence and requests for materials should be addressed to D.A.J. 